\title{
La dotación de interpretación en lengua de señas española para personas sordas en procedimientos judiciales*
}

\author{
The provision of Spanish Sign Language interpreting \\ services for deaf people in legal proceedings
}

Rayco H. González-Montesino ${ }^{1 \varnothing}$

Fecha correspondencia:

Recibido: 19 de abril de 2020.

Revisión: 2 de junio de 2020.

Aceptado: 4 de junio de 2020

Forma de citar:

González, Rayco H. "La dotación de interpretación en lengua de señas española para personas sordas en procedimientos judiciales". En: Revista CES Derecho. Vol. 11, №. 2, junio a diciembre de 2020, 50-69.

Open access

Términos de uso

Licencia creative commons

Etica de publicaciones

Revisión por pares

Gestión por Open Journal System

DOl: http://dx.doi.org/10.21615/

cesder.11.2.4

ISSN: 2145-7719

Sobre el artículo:

El presente artículo retoma y actualiza epígrafes de la tesis doctoral del autor, La estrategia siempre a mano: propuestas didácticas para la interpretación en lengua de signos, defendida en 2016 en la Universidad de Vigo (España) y con la que obtuvo el grado de Doctor cum laude en Lingüística Aplicada.

\section{Resumen}

La interpretación de lenguas de señas es la principal medida para facilitar el acceso a los servicios públicos para las personas sordas españolas. La publicación de la Ley 27/2007, con la que se reconocen estas lenguas y el derecho a su uso, fue un hito para la comunidad sorda. La transposición al ordenamiento jurídico de diversas directivas europeas sobre traducción e interpretación, mediante la Ley Orgánica 5/2015, ha venido a respaldar el derecho de las personas sordas a contar con servicios de interpretación en los diferentes procedimientos judiciales. El presente artículo tiene como objetivo analizar cómo la Administración de Justicia dota de estos servicios de interpretación. Mediante la revisión de la legislación y la comparación con otras experiencias internacionales, hemos detectado las debilidades de nuestro sistema y planteado propuestas de mejora, como la importancia de evaluar las competencias de los intérpretes para garantizar un juicio justo a las personas sordas.

Palabras clave: Lengua de señas, personas sordas, interpretación judicial, contextos jurídicos, acceso a la justicia.

\section{Abstract}

Sign language interpreting is the measure most frequently implemented by Spanish public services in providing access to deaf people. The enactment of the Spanish Act 27/2007, which recognizes sign languages and the right to use them, was a milestone for the deaf community in the country. The transposition of a number of European Guidelines on translation and interpreting into the Spanish legal system, via the Organic Act 5/2015, has strengthened the right of deaf people to interpreting services in different legal proceedings. Against this framework, this article analyzes how the Administration of Justice provides interpreting services to deaf people. Reviewing and comparing the relevant legislation with other international efforts to provide access to interpreters, the weaknesses of the Spanish system will be identified, and suggestions will be made to improve the provision of such services. Suggested improvements include assessing the skills of sign language interpreters to ensure a fair trial for deaf people. 


\section{Sobre el autor:}

1. Profesor visitante del Dpto. Ciencias de la Educación (Métodos de Investigación y Diagnóstico en Educación), Universidad Rey Juan Carlos. raycoh. gonzalez@urjc.es.Doctor cum laude en Lingüística Aplicada (Univ. de Vigo), Ldo. en Antropología Social y Cultural (UNED) y Dipl. en Logopedia (Univ. de La Laguna). Miembro de la Cátedra Institucional Personas con Discapacidad, Accesibilidad e Inclusión y del Grupo consolidado de Investigación en Educación Inclusiva, Personas con Discapacidad y Accesibilidad Universal. https://gestion2.uric.es/pdil ver/raycoh.gonzalez.
Keywords: Sign language, deaf people, legal interpreting, legal contexts, access to justice.

\section{Introducción}

En España, la interpretación de lengua de señas ${ }^{1}$, no se puede considerar una actividad nueva, aunque sí lo es su profesionalización y reconocimiento social y académico. Al igual que en otros países, esta profesión surgió en el seno de la propia comunidad sorda con el objetivo de eliminar las barreras de comunicación y de acceso a la información a las que se enfrentaban a diario sus miembros, y se ha ido desarrollando de forma paralela a la evolución que ha tenido dicha comunidad en nuestra sociedad (De los Santos Rodríguez y Lara Burgos, 2004). El año 1987 supone el inicio de la profesionalización de esta actividad en nuestro país, con la creación del primer Servicio de intérpretes mímicos en Madrid, y 1995 la fecha en la que se decretó una formación oficial para este colectivo. La publicación del Real Decreto 2060/19952 representó el reconocimiento por parte de la Administración, al menos a nivel educativo, de esta profesión y de la necesidad de adquirir una serie de competencias que superaban el simple aprendizaje de esta lengua. Esta formación reglada en el ámbito de la Formación Profesional ha estado vigente hasta el curso 2015-2016. A partir de entonces, todo programa formativo de intérpretes de lenguas de señas en España debe llevarse a cabo en la esfera universitaria, contando en la actualidad con dos únicas propuestas: el Grado en Lengua de Signos Española y Comunidad Sorda de la Universidad Rey Juan Carlos, y el Grado en Traducción e Interpretación, con un itinerario para la lengua de señas catalana (LSC), en la Universidad Pompeu Fabra.

Hoy en día, gracias a la protección que tienen las personas sordas con la Ley $27 / 2007^{3_{3}}$ para utilizar las lenguas de señas españolas en los diferentes contextos sociales, la interpretación de estas se ha convertido en una medida esencial de accesibilidad e igualdad de oportunidades para dicho colectivo. No obstante, tal y como afirma Storch de Gracia y Asensio (2008), las personas sordas y con discapacidad auditiva usuarias de las lenguas de señas han sido "objeto de discriminación y en muchos sentidos sigue[n] siéndolo, tanto en las legislaciones como en la práctica cotidiana, especialmente en todo cuanto se refiere al ejercicio de derechos -incluso fundamentales - que usan el lenguaje como medio de articulación o desarrollo" (p. 2). El presente estudio tiene como objetivo principal analizar cómo es la dotación de servicios de traducción e interpretación para las personas sordas por parte de la Administración de Justicia española para garantizar que disfruten de un juicio justo y una tutela judicial efectiva.

Para poder lograr nuestro propósito, hemos organizado este trabajo en cinco apartados. Comenzamos haciendo una radiografía, apoyada en datos cuantitativos, de la importancia que tiene la interpretación en lengua de señas para el acceso de las personas sordas a los servicios públicos en general. A continuación, presentamos una revisión de cuáles son los principales contextos jurídicos en los que interviene un intérprete de lengua de señas (ILS) para, después de describir el enfoque metodológico utilizado, centrarnos en la dotación de estos profesionales en el ámbito

\footnotetext{
1. Aunque existen suficientes motivos lingüísticos y etimológicos como para utilizar en español el término lengua de señas para denominar al sistema lingüístico utilizado de forma natural por las personas sordas (cfr. Storch de Gracia y Asensio, 1998), en España es habitual emplear la expresión lengua de signos, y es de esta forma como han sido reconocidas legalmente. No obstante, y teniendo en cuenta que en Hispanoamérica es más común el uso de la primera forma, hemos decidido utilizarla de forma preferente en este artículo para facilitar la comprensión de las ideas que en él plasmamos.

2. Real Decreto 2060/1995, de 22 de diciembre, por el que se establece el título de Técnico superior en Interpretación de la Lengua de Signos y las correspondientes enseñanzas mínimas (BOE núm. 47, de 23/02/1996).

3. Ley $27 / 2007$, de 23 de octubre, por la que se reconocen las lenguas de signos españolas y se regulan los medios de apoyo a la comunicación oral de las personas sordas, con discapacidad auditiva y sordociegas (BOE núm. 255, de 24/10/2007)
} 
judicial. Para ello, analizamos la legislación española vinculada a la protección de los derechos de las personas sordas en los procedimientos judiciales y, en concreto, en la regulación de la dotación de servicios de interpretación que garanticen la accesibilidad a estos mediante la transposición al ordenamiento jurídico español de directivas europeas en esta materia. Seguidamente, realizamos un análisis crítico de las condiciones en las que se está aplicando dicha medida en España y cómo puede afectar a las garantías procesales necesarias y esenciales de las personas sordas. Por último, indicamos las principales conclusiones de este estudio, entre las que destaca la necesidad de que Administración de Justicia española establezca un sistema de evaluación que garantice las competencias de los ILS que vayan a desempeñar su labor en los contextos judiciales, así como la incorporación de estos profesionales en el Registro Oficial de Traductores e Intérpretes Judiciales que debe crearse para tal fin.

\section{La interpretación de la lengua de señas para acceder a los servicios públicos}

La interpretación para los servicios públicos se ha consolidado en los últimos años como uno de los tipos de interpretación más importantes para las lenguas orales por los procesos migratorios y las obligaciones administrativas que llevan consigo. Sin embargo, este es un ámbito de interpretación de larga tradición e importancia para las lenguas de señas, aunque el hecho de que la comunidad sorda sea una minoría lingüística y cultural ha supuesto que la labor que desempeñan estos intérpretes pase completamente desapercibida para el conjunto de la sociedad. Las personas sordas requieren del ILS como mediador comunicativo para acceder a los distintos servicios que desde la Administración pública se ponen a disposición de cualquier ciudadano, como la sanidad, la educación, la justicia, los servicios sociales y públicos, etc. Los contextos jurídicos son uno de los principales ámbitos de actuación para los intérpretes de lenguas orales y de señas. La interpretación legal, la jurada y la judicial han adquirido a lo largo de las últimas décadas relevancia no solo a nivel social, sino también se han convertido en objeto de análisis desde los Estudios de Traducción e Interpretación y de formación especializada para traductores e intérpretes. Pöchhacker (2004) afirma que:

In many jurisdictions, what is commonly labeled 'court interpreting' includes tasks like the certified translation of documents as well as interpreting in quasi-judicial and administrative hearings. One can therefore distinguish between the broader notion of legal interpreting, or judicial interpreting, and courtroom interpreting in its specific, prototypical setting. (p. 14)

Estos tipos de mediación comunicativa se dan en sociedades complejas, tanto desde un punto de vista administrativo como social, político, étnico y lingüístico; es decir, se realizan en contextos intra-sociales. Esta labor traslativa consigue eliminar las barreras de comunicación que existen entre personas de una misma población, pero que utilizan lenguas diferentes y pertenecen a otras culturas. Desde la década de los sesenta del pasado siglo, la interpretación de lengua de señas se erigió como uno de los principales referentes en la interpretación en contextos intra-sociales -especialmente en contextos educativos-, ya que el principio de igualdad de todos los ciudadanos empezó a calar profundamente en diversos Estados, desarrollando legislación específica en favor de los colectivos más vulnerables, entre los que se incluyen las personas con alguna discapacidad (Pöchhacker, 2004). 
Sin embargo, como justificaremos con datos a continuación, la contratación directa de profesionales de la interpretación de lengua de señas por parte de la Administración pública española para la realización de su labor en los servicios públicos es hoy en día prácticamente nula o muy escasa. Lo normal es que a estos contextos sociales, administrativos, sanitarios o jurídicos acudan ILS que están contratados por las distintas entidades pertenecientes al movimiento asociativo de las personas sordas. Aparte de las subvenciones para la contratación de ILS de corporaciones locales y de instituciones que administran las distintas comunidades autónomas que integran España, la principal fuente de financiación de este servicio es el Ministerio de Sanidad, Consumo y Bienestar Social -en enero de este año, con el inicio de la presente legislatura, ha pasado a dividirse en tres Ministerios: el de Sanidad, el de Derechos Sociales y el de Consumo-. Desde el año 2002 hasta el 2013 esa financiación de los servicios de interpretación se realizaba mediante la suscripción de un convenio anual entre este Ministerio de Sanidad — con la variedad de nomenclatura que se le ha dado en estos años - y la Confederación Estatal de Personas Sordas (CNSE). A partir del año 2014, la financiación se incluye en las subvenciones estatales concedidas para la realización de programas de interés general. Mediante esta se asegura que las personas sordas puedan ejercer sus derechos básicos y realizar todas las gestiones necesarias para tal fin ante la Administración a través del uso de su lengua natural.

La revista Faro del silencio (CNSE, 2012, pp. 26-32) publicó un reportaje especial por los veinticinco años de la puesta en marcha del primer servicio oficial de ILS en España. En este se hace un análisis de la situación en la prestación de servicios de interpretación en los distintos contextos, incluyendo opiniones de usuarios sordos. Además, ofrece una gráfica en la que se puede observar la evolución en el número de servicios prestados durante los diez años de convenio entre el Ministerio y la CNSE, que logró su máximo histórico en 2007 con un total de 33.934 servicios realizados.

Los últimos datos sobre el número de servicios presenciales prestados por la Red Estatal de Servicios de Interpretación de la CNSE corresponden al año 2018, en el que se realizaron un total de 15.116. Esta cifra es un 22\% mayor a la del año anterior, en el que se alcanzó los 12.422 servicios prestados. Sin embargo, en esa Memoria 2017 de la CNSE se afirma que los "servicios de intérpretes estatales, regionales y locales no cubren la totalidad de las demandas de las personas sordas" (p. 24) y que a estos se suman otros 53.000 realizados con financiación privada. En la Memoria 2018 de esta entidad no se incluyen datos de servicios financiados de otra forma que no sea la pública. No obstante, del total de la financiación pública de la CNSE, sí que destaca el porcentaje que corresponde al convenio de ILS, lo que refleja la importancia que tiene este servicio para el colectivo de personas sordas. Así, más de un $62 \%$ de la financiación pública que recibió esta entidad para el año 2018 provino del Ministerio de Sanidad, Consumo y Bienestar Social y sus diferentes organismos, de la que un $38,22 \%$ se destinó exclusivamente a cubrir la interpretación de lengua de señas para los servicios públicos.

Sin embargo, una cuestión preocupante es el número de ILS contratados para desempeñar esta labor y facilitar la accesibilidad en los distintos ámbitos sociales y, sobre todo, el tipo y duración de la contratación de estos profesionales. Según Bao Fente y González-Montesino (2013, p. 299), en el año 2010 la Red Estatal de Servicios de Interpretación de la CNSE estaba compuesta por un total de 121 ILS para un programa semestral, muchos de los cuales son sustituidos por otros profesionales al finalizar dicho programa por la promoción de acceso al primer empleo que las 
Administraciones públicas suelen incluir entre las condiciones de las subvenciones que permiten su contratación. Esto podría repercutir de forma directa en la calidad de la interpretación que se presta para los servicios públicos, ya que la experiencia adquirida durante ese breve periodo de tiempo por parte de estos profesionales de la interpretación termina perdiéndose y los nuevos ILS deberán aprender otra vez el vocabulario utilizado, los temas que se suelen tratar, los guiones comunicativos que se suelen dar en estas situaciones comunicativas, los objetivos y expectativas que suelen tener los interlocutores, etc.

Para completar esta radiografía sobre la interpretación en lengua de señas para los servicios públicos en España, y antes de adentrarnos en el análisis de la labor de estos profesionales en los contextos jurídicos, es necesario hacer referencia también a los servicios de videointerpretación que desde el año 2007 se han implantado en nuestro país mediante la plataforma SVIsual. A través de esta, las personas sordas pueden acceder a servicios de interpretación de lengua de señas desde cualquier lugar del territorio nacional sin desplazamientos y en tiempo real, lo que facilita el acceso a la información y la comunicación, amén de mejorar la calidad de vida de este colectivo. Con una cobertura de las 24 horas de los 365 días del año, gracias al apoyo del Ministerio de Sanidad, Consumo y Bienestar Social y de la Fundación Vodafone España, en el año 2018 se registraron un total de 100.926 llamadas, de las que fueron atendidas un $71 \%$ y que supuso un incremento del $5 \%$ con respecto al año anterior (cfr. CNSE, Memoria 2018, p. 45).

\section{El intérprete de lengua de señas en los principales contextos jurídicos}

Como cualquier otro ciudadano, las personas sordas usuarias de lenguas de señas son partícipes a diario de muchos escenarios comunicativos en los que el discurso jurídico está presente, como por ejemplo un acto notarial para la compraventa de un bien inmueble, la firma de un contrato de alquiler de una vivienda, la transmisión de una herencia en la lectura de un testamento, la realización de una denuncia en una comisaría de policía, la declaración como testigo en un juicio, etc. En todas ellas, la presencia de un ILS como mediador interlingüístico e intercultural se hace imprescindible, tanto para que la(s) persona(s) sorda(s) pueda(n) expresar y comprender lo que se dice a través de su lengua natural, como para que la(s) persona(s) oyente(s) logre(n) transmitir sus enunciados y entender los que seña(n) su/(s) interlocutor(es). En este apartado, y teniendo en cuenta que el número de situaciones comunicativas, participantes y roles asumidos por estos en el ámbito jurídico es tan amplio que es prácticamente inabarcable en cualquier estudio, vamos a tratar de describir algunos de los principales escenarios en los que un ILS se verá involucrado.

Si tenemos en cuenta la realidad socioeconómica y educativa de las personas sordas en España, que se caracteriza por un alto índice de abandono escolar y desempleo, empleos de baja categoría profesional y mal remunerados, así como una deficiente formación académica con dificultades para el acceso a la educación secundaria y superior ${ }^{4}$ (Acosta Rodríguez, 2006), la imagen de los participantes que puede tener un ILS cuando se le solicita mediar en un servicio jurídico es la de una persona sorda que, por algún motivo, debe comunicarse con una o varias personas oyentes que, o bien forman parte de la Administración de Justicia, o son miembros de las Fuerzas

4. El acceso, permanencia y egresión del alumnado sordo y con discapacidad auditiva de las universidades españolas es realmente escasa. Así, por ejemplo, encontramos que en 1999 solo un 28\% de la población sorda había terminado estudios universitarios (CNSE 2003) y que en el curso 2006-2007 el $7,8 \%$ de los estudiantes con discapacidad matriculados en estudios universitarios presentaba una discapacidad auditiva (Red2Red Consultores, 2009). 
y Cuerpos de Seguridad del Estado, o ejercen el Derecho de forma privada, etc. El ámbito de la interpretación legal o jurídica es en sí mismo muy amplio, tanto como lo son las distintas ramas y especialidades del Derecho, por lo que el ILS deberá tener amplios conocimientos sobre esta temática y esta lengua de especialidad para poder realizar una labor eficaz.

Hale (2010) señala que los principales ámbitos jurídicos del mundo angloparlante donde se requiere la presencia de un intérprete de lenguas orales son: interrogatorios y entrevistas policiales, interacción entre abogado y cliente, vistas en juzgados de rango inferior, vistas y juicios en tribunales. De acuerdo con De los Santos Rodríguez y Lara Burgos (2004), los servicios jurídicos en los que debe participar un ILS "comprenden todas aquellas situaciones de interpretación que se desarrollan sobre temas legales, tales como entrevistas con abogados, notarios, declaraciones en comisarías, detenciones, juicios y trámites de toda índole" (p. 154). Napier, McKee y Goswell (2010, p. 125) también incluyen los centros penitenciarios entre los contextos en los que puede ser necesaria la presencia de un ILS. Estas autoras afirman que este profesional será contratado en el ámbito Penal cuando una persona sorda haya sido arrestada por alguna causa, sea víctima o acusada de un delito, o actúe como testigo; y en el ámbito Civil cuando la persona sorda sea parte de un caso en un juzgado de lo Civil o en un juzgado de Familia, así como en consultas a abogados. Storch de Gracia y Asensio (2008) hace un análisis muy completo de las diferentes fases de la actuación notarial cuando participan personas sordas o con discapacidad auditiva, concretando los ajustes razonables a adoptar en estos casos y entre los que se encuentra, de forma prioritaria, la intervención de un ILS.

Napier (2013) señala, además, otras dos situaciones jurídicas emergentes en algunos países en los que el ILS es necesario: cuando la persona sorda es abogada y requiere del profesional en consultas de sus clientes o durante un juicio, o cuando la persona sorda actúa como miembro de un jurado popular. Para este último caso, Napier, Spencer y Sabolcec (2007) realizaron un estudio con el objetivo de evaluar la precisión en la interpretación de conceptos jurídicos del inglés a la Lengua de Señas Australiana (Auslan), así como determinar el nivel de comprensión de la información al que llegaban los participantes: un jurado formado por seis personas oyentes y seis sordas. Para ello, analizaron las interpretaciones de un simulacro de juicio realizadas por una pareja de ILS con experiencia en el ámbito. También realizaron una prueba de comprensión y unas entrevistas post-test a los participantes que actuaban como jurado. Encontraron que los conceptos y términos legales de los dos textos interpretados al Auslan alcanzaron un $87,5 \%$ de exactitud, y que tanto los jurados sordos como los oyentes lograron un alto nivel de comprensión de estos - un $75 \%$ y un $78 \%$ respectivamente-, lo que no supone diferencias significativas entre ambos grupos. Las principales conclusiones a las que llegaron son:

1. legal facts and concepts can be translated into Auslan;

2. Auslan interpreting can provide effective access to court proceedings for a deaf juror - but certain conditions are necessary;

3. hearing people misunderstand court proceedings without being disadvantaged by hearing loss; and

4. deaf people are willing and able to serve as jurors. (Napier et al., 2007, p. 62).

Parte de este estudio fue replicado en diversas ciudades australianas con un número mayor de participantes - treinta oyentes y treinta sordos-con el objetivo de 
determinar la fiabilidad de los resultados y si aparecía alguna diferencia significativa desde el punto de vista estadístico. Los resultados corroboraron que tanto las personas sordas como las oyentes no entienden por completo las conclusiones del juez en un proceso judicial, y no se observaron diferencias significativas en cuanto a edad, género o empleo, pero sí en los estudios cursados (Napier, 2013, pp. 8-9).

Por último, debemos destacar el reciente estudio realizado por Napier y Haug (2017) como parte del proyecto europeo Justisigns ${ }^{5}$, que nos ofrece una amplia panorámica de la situación de la interpretación de las lenguas de señas en los contextos jurídicos en el continente europeo. En concreto, y en consonancia con lo apuntado en este apartado, estos autores encuentran que la interpretación en lengua de señas "is most commonly provided in legal settings where deaf people are involved as complainants, defendants, or witnesses, rather than for deaf people serving as jurors" (p. 6). En menor medida, también se facilita este servicio en notarías, protección de menores, tribunales y prisiones.

\section{Propuesta metodológica}

El objetivo principal de este trabajo es analizar las fortalezas y debilidades en la dotación de servicios de interpretación en lengua de señas por parte de la Administración de Justicia española, para proponer posibles mejoras que reviertan en una mayor garantía de los derechos de las personas sordas a la hora de disfrutar de un juicio justo. En concreto, nos interesa conocer cómo se regula la provisión de estos profesionales en los contextos judiciales y de qué forma se están implementando las directivas europeas en relación con la traducción e interpretación.

Desde un enfoque multidisciplinar y novedoso en el panorama español, que incluye al Derecho, a los Estudios de Interpretación y a los denominados Deaf Studies, realizaremos una revisión de la legislación española vinculada con los derechos de accesibilidad e igualdad de oportunidades de las personas sordas, analizando en profundidad los artículos que regulan y garantizan la dotación de servicios de traducción e interpretación en lengua de señas como medida de accesibilidad a los contextos judiciales. Utilizamos para ello el método dogmático, ya que el fundamento de este estudio se encuentra en las normas jurídicas y su análisis será abstracto o teórico (Tantaleán Odar, 2016). No obstante, coincidimos con Sarlo (2006) en que esta perspectiva dogmática que aplicaremos "no es meramente descriptiva, porque su interés práctico supone necesariamente valoraciones y finalidades" (p. 177). Tales valoraciones nos permitirán concretar el grado y modo de cumplimiento de la normativa que debe garantizar que las personas sordas señantes disfruten de procedimientos equitativos.

Se trata de un estudio preliminar en el que la técnica de investigación empleada es la documental, mediante la recopilación y análisis de fuentes primarias y secundarias: documentos normativos y legislativos, directivas, informes, artículos científicos, etc. Para la selección de estas fuentes utilizamos palabras clave en español e inglés y diferentes operadores booleanos en bases de datos especializadas, como ERIC, Google académico o Dialnet, así como en el motor de búsqueda del Boletín Oficial del Estado. 


\section{La interpretación de la Lengua de Señas Española en los procedimientos judiciales}

El primer texto al que debemos hacer alusión es, lógicamente, la Constitución española $(1978)^{6}$. En su artículo 9.2 se señala la responsabilidad de los poderes públicos a la hora de que cualquier ciudadano participe plenamente en la sociedad, eliminando para ello cualquier obstáculo que lo impida o dificulte. Además, en materia de discapacidad es esencial el artículo 49, ya que salvaguarda los derechos de estas personas e indica que se les debe prestar la atención especializada que requieran. Por supuesto hay que hacer referencia al artículo 14, que reconoce que todo ciudadano español es igual ante la ley y que no puede ser discriminado por razón alguna, y también a los artículos 17.3 y 24.1 porque garantizan, respectivamente, que cualquier detenido sea informado de sus derechos y los motivos de su detención de forma comprensible, y el derecho a una tutela judicial efectiva.

En nuestro país encontramos un amplio marco legislativo en materia de discapacidad y accesibilidad, de carácter tanto nacional como autonómico, con el que se garantizan estos derechos constitucionales. La Ley 13/1982, de 7 de abril, de integración social de los minusválidos ${ }^{7}$-en términos de esa época- vino a desarrollar el artículo 49 y fue una ley esencial en el reconocimiento social de los derechos de las personas con discapacidad. Sus principios de respeto a este colectivo y vocación de integración social se incorporaron a leyes en materia de educación, salud o empleo. Un paso más fue la promulgación de la Ley 51/2003, de 2 de diciembre, de Igualdad de oportunidades, no discriminación y accesibilidad universal de las personas con discapacidad ${ }^{8}$. En ella se establecieron medidas concretas que garantizaran la accesibilidad de todas las personas, sin discriminación alguna, a los diferentes contextos sociales, entre los que se incluía la Administración de Justicia. Entre dichas medidas estaba la dotación de servicios personales de apoyo a la comunicación en lengua de señas (art. 10.2.c). Hoy día, ambas leyes están derogadas e integradas, junto a la Ley 49/20079, en el Real Decreto Legislativo 1/2013, de 29 de noviembre, por el que se aprueba el Texto Refundido de la Ley General de derechos de las personas con discapacidad y de su inclusión social ${ }^{10}$. En este último también se señala que, con el fin de garantizar unas condiciones básicas de accesibilidad y no discriminación a las personas con discapacidad en diferentes ámbitos -incluido el de la Administración de Justicia-, se establecerán unas medidas concretas. Entre estas está el ILS, como servicio de apoyo personal para la comunicación (art. 23.2.c).

Naturalmente, la Ley 27/2007 es actualmente el principal instrumento que utilizan las personas sordas para reclamar sus derechos ante la Administración pública en relación con el uso de la lengua de señas, "a pesar de tratarse de una ley que en la actualidad no está plenamente desarrollada" (Báez Montero y Fernández Soneira, 2015, p. 125). Es más, en estos últimos años encontramos manifestaciones que defienden la equiparación de las lenguas de señas españolas al resto de lenguas cooficiales del Estado y, por tanto, su mención constitucional expresa.

6. BOE núm. 311, de 29/12/1978. (Consultado el 4 de abril de 2020)

7. BOE núm. 103, de 30/04/1982. (Consultado el 5 de abril de 2020).

8. BOE núm. 289, de 03/12/2003. (Consultado el 5 de abril de 2020).

9. Ley 49/2007, de 26 de diciembre, por la que se establece el régimen de infracciones y sanciones en materia de igualdad de oportunidades, no discriminación y accesibilidad universal de las personas con discapacidad. (BOE núm. 310, de 27/12/2007). (Consultado el 5 de abril de 2020).

10. BOE núm. 289, de 03/12/2013. (Consultado el 5 de abril de 2020). 
Belda Pérez-Pedrero (2016), por ejemplo, argumenta los motivos jurídicos por los que propone una adenda al artículo 3.2 cuando nuestra Carta Magna sea reformada, y considera que:

Reflejando al máximo nivel normativo la realidad de uso de la lengua de signos, se efectúa una remoción de obstáculos para la integración de las mismas [de las personas sordas], se previenen afecciones a la igualdad en esta categoría de personas susceptibles de discriminación, y se posibilita el ejercicio pleno de todos y cada uno de sus derechos constitucionales. (p. 117)

No obstante, hasta que esto pase - si es que ocurre en algún momento-, se debe seguir haciendo uso y reclamando lo establecido en la Ley 27/2007 en relación con la interpretación en lengua de señas. Así, el artículo 9 encarga a los poderes públicos a que promuevan la prestación de servicios profesionales de interpretación en diferentes ámbitos públicos y privados, entre los que incluye las relaciones con la Administración de Justicia y Penitenciaria. Esta debe impulsar la formación y disponibilidad de estos profesionales "para hacer efectiva la aplicación de lo dispuesto en el artículo 143 de la Ley de Enjuiciamiento Civil, así como en los procesos que se rigen por la Ley de Enjuiciamiento Criminal, respecto de las personas sordas, con discapacidad auditiva y sordociegas" (art. 12.2).

El artículo 143.2 de la Ley 1/2000, de 7 de enero, de Enjuiciamiento Civil, ${ }^{11}$ señala que "si la persona fuere sorda, se nombrará siempre [...] al intérprete de lengua de signos adecuado" y que se levantará acta de estas actuaciones. Por su parte, la Ley de Enjuiciamiento Criminal (LECrim) ${ }^{12}$ indica que se nombrará un ILS cuando un testigo sordo tenga que realizar una declaración, y que dicho intérprete debe prestar juramento previo en presencia de este testigo (art. 442). Además, el ILS tendrá que leerle esa declaración al testigo sordo (art. 443).

La LECrim y la Ley Orgánica 6/1985, de 1 de julio, del Poder Judicial ${ }^{13}$ han sido modificadas parcialmente con la promulgación de la Ley Orgánica 5/201514, de 27 de abril -que será el texto legislativo que utilizaremos en los siguientes apartados para analizar en profundidad cómo es la dotación de servicios de interpretación de lengua de señas en contextos judiciales-, y que realiza la transposición a nues-

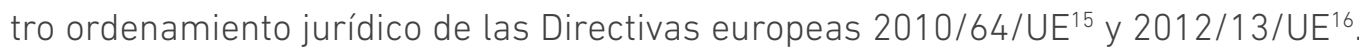
Estas son dos de las cuatro ${ }^{17}$ directivas elaboradas por el Parlamento Europeo y el

11. BOE núm. 7. de 08/01/2000. (Consultado el 7 de abril de 2020)

12. Real Decreto de 14 de septiembre de 1882 por el que se aprueba la Ley de Enjuiciamiento Criminal (BOE núm. 260, de 17/09/1882). (Consultado el 7 de abril de 2020)

13. BOE núm. 157, de 02/07/1985. (Consultado el 7 de abril de 2020)

14. Ley Orgánica 5/2015, de 27 de abril, por la que se modifican la Ley de Enjuiciamiento Criminal y la Ley Orgánica 6/1985, de 1 de julio, del Poder Judicial, para transponer la Directiva 2010/64/UE, de 20 de octubre de 2010, relativa al derecho a interpretación y a traducción en los procesos penales y la Directiva 2012/13/UE, de 22 de mayo de 2012, relativa al derecho a la información en los procesos penales (BOE núm. 101, de 28/04/2015). (Consultado el 7 de abril de 2020)

15. Directiva 2010/64/UE del Parlamento Europeo y del Consejo, de 20 de octubre de 2010, relativa al derecho a interpretación y a traducción en los procesos penales. (DOUE L 280, de 26/10/2010). (Consultado el 7 de abril de 2020).

16. Directiva 2012/13/UE del Parlamento Europeo y del Consejo, de 22 de mayo de 2012, relativa al derecho a la información en los procesos penales (DOUE L 142, de 01/06/2012). (Consultado el 7 de abril de 2020).

17 Las otras dos son la Directiva 2012/29/UE, de 25 de octubre de 2012, por la que se establecen normas mínimas sobre los derechos, el apoyo y la protección de las víctimas de delitos (DOUE L 315, de 14/11/2012). y la Directiva 2013/48/UE, de 22 de octubre de 2013, sobre el derecho a la asistencia de letrado en los procesos penales y en los procedimientos relativos a la orden de detención europea, y sobre el derecho a que se informe a un tercero en el momento de la privación de libertad y a comunicarse con terceros y con autoridades consulares (DOUE L 294, de 06/11/2013). 
Consejo, según lo dispuesto en el Tratado de Funcionamiento de la Unión Europea ${ }^{18}$, que garantiza en el orden penal a toda persona a disfrutar de un juicio justo, mediante servicios de traducción e interpretación.

De entre ellas, queremos destacar la Directiva 2010/64/UE, de 20 de octubre de 2010. Este documento determina un conjunto de normas mínimas en materia de traducción e interpretación que todos los Estados miembros deben tener presentes para asegurar el derecho a defensa y a un juicio justo a cualquier persona, sospechosa o acusada de un delito, que no hable o comprenda la lengua utilizada en dicho procedimiento penal. El fin último de este texto es que haya una prestación de servicios de intermediación lingüística de calidad a lo largo de todo el proceso penal, incluyendo el interrogatorio policial o las vistas previas necesarias. Esto supone, entre otras cosas, la gratuidad y pronta prestación del servicio, el derecho a que las conversaciones entre el sospechoso o acusado y su abogado sean interpretadas, el derecho a la traducción de documentos esenciales, etc. Además, un aspecto destacable para nuestro trabajo es que el artículo 2.3 hace referencia explícita a que este derecho también recae en las personas con discapacidad auditiva.

Una vez hemos descrito los aspectos más relevantes de esta Directiva 2010/64/UE y referido lo que establece la legislación española en cuanto al derecho de cualquier persona a poder disfrutar de un juicio justo y, en el caso de las personas sordas, a proporcionarles servicios de interpretación en lengua de señas para garantizar su accesibilidad en estos contextos en igualdad de condiciones, pasamos a continuación a analizar cómo se está aplicando la citada Ley Orgánica 5/2015. Según Ibáñez Castaño (2019), la aprobación de esta ley ha supuesto "un avance en los derechos de las personas sordas o con discapacidad auditiva a un juicio equitativo y a una tutela judicial efectiva" (p. 727). En general, estamos de acuerdo con esta afirmación, aunque se hace necesario valorar la aplicación de algunos artículos de la citada norma y comparar la situación de la interpretación en lengua de señas en otros países del mundo con más experiencia que el nuestro o con la situación de los traductores e intérpretes de lenguas orales en el ámbito judicial español.

\section{Análisis crítico de la aplicación de Ley Orgánica 5/2015 en relación con la prestación de servicios de interpretación de Lengua de Señas Española en contextos judiciales}

\section{¿Quién puede rehusar del intérprete de lengua de señas asignado y solicitar uno nuevo?}

Una de las modificaciones en la LECrim que supone la publicación de la Ley Orgánica 5/2015 se da en el artículo 124.3. Este precepto plantea una cuestión que, posiblemente, sea la que más recelo pueda generar en el colectivo de profesionales de la interpretación de lenguas de señas en España. Dicho artículo da la potestad al imputado o acusado sordo de poder solicitar la designación de un nuevo ILS si aprecia que su labor no está ofreciendo garantías suficientes de exactitud. Sin embargo, en el caso de los traductores e intérpretes de lenguas orales esa opción solo está en disposición del Tribunal, el Juez o el Ministerio Fiscal, de oficio o a instancia de parte, y siempre tras realizar las comprobaciones que se consideren oportunas. Autores como Brunson (2008) también consideran que "(a)s knowledgeable agents,

18. Según el artículo 82.2. el Parlamento Europeo y el Consejo podrán establecer normas mínimas mediante directivas que faciliten el reconocimiento mutuo de las sentencias y resoluciones judiciales y la cooperación policial y judicial en asuntos penales con dimensión transfronteriza. Recuperado de https://www.boe.es/doue/2010/083/Z00047-00199.pdf 
deaf people must be able to determinate not only whether an interpreter should be provided but also which interpreter" (p. 90).

Sin embargo, esta medida supone una diferencia de trato hacia el colectivo de personas sordas en comparación con otros acusados que no dominan la lengua en la que se desarrolla el procedimiento. Debemos tener presente que, en muchas ocasiones, la preferencia que tienen las personas sordas por un ILS u otro no se relaciona con sus habilidades o competencias profesionales, sino con la personalidad de estos o afinidad (cfr. Brunson, 2008, p. 90). En cierta medida, para las lenguas de señas y las personas sordas, la Administración de Justicia española está haciendo dejación de su responsabilidad en cuanto a dotar de servicios de traducción e interpretación de calidad que garanticen una accesibilidad en igualdad de condiciones y un juicio justo. Es más, la propia LECrim establece que "[e]l traductor o intérprete judicial será designado de entre aquellos que se hallen incluidos en los listados elaborados por la Administración competente" (art. 124.1). Es decir, es la propia Administración de Justicia la que debe establecer los procedimientos para asegurar la disponibilidad de traductores e intérpretes con las cualificaciones y competencias necesarias para desempeñar una labor adecuada y con precisión, independientemente de la lengua de la que se trate (Ley Orgánica 5/2015, disp. final primera). Con ese listado o Registro Oficial de Traductores e Intérpretes Jurados no se pondría en riesgo la celebración del procedimiento, además de que se protegería al imputado o acusado sordo y se evitaría trasladarle una responsabilidad que no le corresponde, sobre todo en una situación que de por sí es estresante para cualquier persona (Hale, 2010).

\section{¿Qué tipo de cualificaciones deben tener los intérpretes de lengua de señas en los ámbitos judiciales y cómo se evalúan?}

Desde el ámbito profesional y académico se ha puesto de manifiesto en reiteradas ocasiones $^{19}$ la necesidad de establecer un sistema de evaluación objetivo y fiable — similar al que se utiliza con los Traductores e Intérpretes Jurados- para aquellos aspirantes a participar en el Registro Oficial de Traductores e Intérpretes Judiciales que debía crearse, en el plazo máximo de un año, a raíz de la Ley Orgánica $5 / 2015^{20}$. El Ministerio de Asuntos Exteriores y de Cooperación (MAEC), así como las comunidades autónomas con competencia en esta materia, habilita y nombra como Intérprete y Traductor Jurado ${ }^{21}$ a todo aquel que supere los exámenes ${ }^{22}$ que el propio Ministerio convoca anualmente, que pueda acreditar la realización de un número determinado de créditos en esta temática mientras cursaba la

19. La Red Vértice de asociaciones de traductores, correctores e intérpretes, la Asociación Profesional de Traductores e Intérpretes Judiciales y Jurados (APTIJ) y la Conferencia de Centros y Departamentos Universitarios de Traducción e Interpretación (CCDUTI) publicaron diversas notas de prensa y comunicados durante el proceso de aprobación de la Ley Orgánica 5/2015, en las que manifestaban su postura ante la misma. La última nota fue el 17 de abril de 2015, al día siguiente de la publicación en BOE de la Ley, bajo el título "Oportunidad perdida: interpretación judicial sin garantías". Recuperado de http:// www.aptij.es/img/doc/Nota\%20de\%20prensa\%2017-04-2015.pdf

20. Según denuncia ARTIJ, a fecha de 14 de marzo de 2019 sigue sin haberse creado este Registro Oficial de Traductores e Intérpretes Judiciales, aunque se han sucedido en el cargo distintos equipos de gobierno en los últimos años. Recuperado de http://www.aptij.es/img/doc/nota\%20aptij\%20iv\%20 convenio\%20END.pdf

21. Cabe destacar que, aunque las lenguas de señas españolas están reconocidas por Ley desde el año 2007, hasta el momento no se ha contemplado la posibilidad de incorporarlas a los exámenes conducentes al título de Intérprete y Traductor Jurado, a diferencia de lenguas orales en desuso como puede ser el latín —en la convocatoria de exámenes del 2015, disponible en https://www.boe.es/boe/dias/2015/01/27/pdfs/B0E-A-2015-692.pdf-, aun cuando muchas personas sordas y oyentes se beneficiarían de contar con profesionales acreditados en traducción e interpretación de lenguas de señas para participar en diversos actos y procedimientos.

22. Ortega Herráez (2011) realiza un análisis comparativo de las pruebas utilizadas en distintos años para la acreditación de intérpretes y traductores jurados del MAEC y de la Generalitat de Cataluña, así como para traductores e intérpretes jurídicos en plantilla. Entre las conclusiones a las que llega el autor, destaca el que "a pesar de que todos los sistemas analizados tienen como objetivo la acreditación de profesionales que desempeñen tareas de interpretación en entornos jurídicos, salvo en un caso, ninguno cuenta entre sus pruebas con ejercicios específicos destinados a evaluar la competencia en interpretación de los candidatos" (p. 147). Se considera suficiente para tal fin una prueba de competencia lingüística. Esto queda patente en la última convocatoria de examen para la obtención del título de Traductor-Intérprete Jurado, del año 2018, en la que las tres pruebas que comprenden el segundo ejercicio evalúan exclusivamente la competencia traductológica. Recuperado de http://www.exteriores.qob.es/Portal/es/ServiciosAlCiudadano/OportunidadesProfesionalesFormacion/OportunidadesProfesionales/traduccioneinterpretacion/Documents/BOE-A-2018-9989.pdf 
licenciatura de Traducción e Interpretación, o mediante el reconocimiento de cualificaciones profesionales análogas obtenidas en otro Estado miembro de la Unión Europea o del Espacio Económico Europeo. Se garantiza así que el profesional posee las destrezas, competencias y cualificaciones académicas necesarias para desempeñar esta labor tanto en el ámbito público como privado ${ }^{23}$. Sin embargo, para la inscripción en el futurible Registro Oficial de Traductores e Intérpretes Judiciales, el Ministerio de Justicia podrá determinar para algunas lenguas otros requisitos diferentes a la formación o titulación reglamentaria, "y podrán basarse en la experiencia del profesional, en el conocimiento adicional de cuestiones procedimentales o jurídicas, y en el cumplimiento de deberes deontológicos previstos en la ley" (Ley Orgánica 5/2015, Disposición final primera).

En algunos países es frecuente la realización de pruebas para la evaluación de competencias y la existencia de sistemas de acreditación de los ILS para los distintos ámbitos de trabajo, entre ellos el jurídico, lo que garantiza la calidad en la prestación de servicios (Napier, 2004). Estamos de acuerdo con el Registro de Intérpretes para Sordos (Registry of Interpreters for the Deaf [RID]: 2007) en que "(l)egal interpreting requires highly skilled and trained specialists because of the significant consequences to the people involved in the event of a failed communication" (p. 1). Esta organización acredita desde 1998 a todos aquellos ILS de Estados Unidos que demuestren, mediante examen, tener los conocimientos jurídicos y las habilidades necesarias para este tipo de escenarios, certificado que debe ser renovado cada cinco años ${ }^{24}$.

En Europa, Napier y Haug (2017) encuentran que "more than 50 percent of the country respondents do not require specific legal interpreting certification for sign language interpreters; more than 50 percent do not have quality assurance processes for legal SLI" (p. 11). Un ejemplo de ello lo encontramos en España, ya que a los ILS que desempeñan labores en contextos jurídicos no se les exige ningún tipo de especialización ${ }^{25}$; hasta el momento, solo es necesario que tengan el título de Técnico Superior o que documenten su condición como tal, lo que no garantiza que dicho profesional pueda realizar con eficacia esta labor. Se hace necesario, por tanto, que se aplique lo que señala la Resolución 2016/2952 del Parlamento Europeo sobre especialización y cualificación de los ILS en contextos judiciales y coincidimos en los planteamientos que hacen a este respecto Storch de Gracia y Asensio (2008) y Paz Arranz (2009, citado en Ibáñez Castaño, 2019, p. 745).

De Wit y Salami (2012) afirman que en ningún país europeo existe un registro exclusivo de ILS que trabajen en contextos jurídicos; en aquellos pocos países en los que ILS están incluidos en registros nacionales de intérpretes lo hacen junto a los de lenguas orales. Las autoras también apuntan que los registros son solo uno de los medios para localizar ILS y que, dependiendo del país, las comisarías o juzgados deben acudir a las agencias o empresas de intérpretes que hayan ganado el concurso público para la dotación de servicios de interpretación, a las asociaciones de

23. Estos profesionales son inscritos de oficio en el Registro de Traductores/as-Intérpretes Jurados/as del MAEC con un número determinado, y anualmente se publica en la web del Ministerio un listado de los que están en activo, las lenguas para las que están habilitados y una forma de contacto. El último listado de 2019 está disponible en: http://www.exteriores.gob.es/Portal/es/ServiciosAlCiudadano/Documents/Listado\%20actualizado.pdf

24. RID (2007, p. 1) afirma que, aunque ha aumentado el número de ILS con certificado de especialista en ámbito legal, la gran demanda que existe hace que en muchos casos este tipo de interpretación las realicen profesionales con certificado de ILS generalista que han recibido algo de formación en este ámbito. Brunson (2008, p. 80) también señala la existencia de ILS sin certificación del RID que a veces realizan labores en procedimientos legales, e incluso la disparidad de habilidades entre ILS que poseen la certificación del RID, cuando estas deberían ser similares.

25. El único programa formativo reglado que se ha realizado en nuestro país con un itinerario específico en la interpretación jurídica para las lenguas de señas fue el Máster Oficial en Docencia e Interpretación en Lengua de Señas de la Universidad de Valladolid, coordinado por D. Carlos Moriyón Mojica y que actualmente no se oferta, que tenía como uno de sus objetivos "el desarrollo de destrezas interpretativas —conceptuales y léxicas- que aseguren a los sordos la igualdad, la integración y la no discriminación, permitiéndoles el acceso a los valores de libertad y de seguridad jurídica". Información recuperada de: http://www.feyts.uva.es/?q=node/34 
personas sordas, a ILS que trabajan por cuenta propia, a listados de intérpretes jurados o cualificados, e incluso a familiares o conocidos de las personas sordas. En nuestro país se pueden dar todas estas situaciones, aunque lo más común es que se solicite el servicio de interpretación a la empresa adjudicataria del concurso, a entidades representativas de personas sordas o a ILS que trabajan como autónomos por cuenta propia y que están incluidos en listas elaboradas a tal fin por la Administración.

\section{¿Cómo se contratan los servicios del intérprete de lengua de señas y qué conse- cuencias tiene la forma en que se hace?}

El artículo 1 de la Ley Orgánica 5/2015 establece que la Administración sufragará los gastos de traducción e interpretación derivados del derecho que tiene el imputado o acusado a ser asistido por un intérprete en todas las actuaciones en las que sea necesaria, incluyendo el interrogatorio policial o por el Ministerio Fiscal, todas las vistas judiciales, las comunicaciones con su abogado a fin de preparar la defensa para cualquier interrogatorio o vista judicial durante el proceso, o para la presentación de un recurso u otras solicitudes procesales cuando sea necesario. Aunque en muchos casos la persona sorda realiza gestiones de forma particular para poder disponer de un ILS en el procedimiento — posiblemente por la costumbre que tiene de solicitarlo para cualquier ámbito de su vida diaria—, es la Dirección General para el Servicio Público de Justicia la encargada de "(l)a programación y coordinación de actuaciones sobre dotación de recursos humanos de los órganos judiciales, Ministerio Fiscal y medicina forense" (Real Decreto 453/2020, art. 4.1.f) $)^{26}$. Esta Dirección es la responsable de la búsqueda, dotación y costeo del ILS. No obstante, en los últimos años ${ }^{27}$ también encontramos que, tanto para los intérpretes de lenguas orales como de señas,

[l]a Administración de Justicia se ha inclinado por subcontratar a empresas privadas los servicios de traducción e interpretación para los juzgados. Se trata de un hecho que ha provocado un incremento del coste de la prestación y que, de forma adicional, ha ido en detrimento de la calidad del servicio. (Gascón Nasarre, 2011, p. 37)

En el caso del Cuerpo Nacional de Policía, la División Económica y Técnica ha optado igualmente por externalizar el servicio de interpretación y traducción de lenguas ${ }^{28}$, por lo que cuando una comisaría requiere de un ILS lo solicita directamente a la empresa adjudicataria del concurso. Coincidimos con Gascón Nasarre (2011) en que este tipo de prácticas resta calidad a los servicios de interpretación, ya que estas empresas, en muchas ocasiones y con el fin de abaratar costes y maximizar beneficios, optan por contratar a intérpretes sin experiencia o sin formación específica para contextos jurídicos y policiales, u ofertan precios irrisorios a intérpretes

26. BOE núm. 63, de 12/03/2020. (Consultado el 9 de abril de 2020).

27. En este enlace podemos ver un ejemplo de 2018 del anuncio de formalización de contratos externos por parte del Ministerio de Justicia para la prestación de servicios de traducción e interpretación en órganos judiciales: https://www.boe.es/boe/dias/2018/07/13/pdfs/B0E-B-2018-37466.pdf

28. En el siguiente enlace se encuentra una Resolución de adjudicación de contratos, con fecha de 7 de febrero de 2019, de diversos lotes de servicios de interpretación de declaraciones orales, traducciones escritas en lenguas extranjeras y cooficiales de España distintas del idioma castellano y lenguaje de signos, tanto de forma directa como inversa, en el marco de las actuaciones policiales en el ámbito de todo el territorio nacional: https://contrataciondelestado.es/wps/wcm/connect/8cd8ef7f-e8fa-49b7-abe8-ad39555cc726/DOC20190226112057RESOLUCIONES+ADJUDICACION.pdf?MOD=AJPERES Llama la atención el uso erróneo que se hace en la convocatoria del término lenguaje de signos, lo que demuestra el grado de conocimiento que se tiene en este ámbito sobre esta materia y sobre la labor de los profesionales vinculados a la misma. 
profesionales ${ }^{29}$. De Wit y Salami (2012) concluyen que es fundamental una remuneración apropiada de los ILS profesionales formados para este tipo de contextos, ya que, por un lado, garantiza unos altos estándares profesionales y, por el otro, anima a que los ILS con experiencia continúen realizando esta labor y no la abandonen, así como que se incorporen nuevos ILS cualificados para este ámbito.

Brunson (2008) afirma que el hecho de que la Administración sea quien costee al ILS hace que las personas sordas no tengan información suficiente de cómo se ha seleccionado al profesional y organizado el servicio. Esto puede suponer, en muchas ocasiones, una adaptación parcial en la eliminación de las barreras de comunicación por las habilidades que realmente tiene el ILS, más allá del título o acreditación que posea, el número de ILS contratados, los conocimientos o familiaridad que tengan con el caso, etc. Como consecuencia, "(s)ometimes, deaf persons are made to feel that they are being done a favor or that they are ungrateful for not accepting the accommodation that is provided and paid for by another" (ibid., p. 82).

Una cuestión que no podemos dejar de comentar es que la simple provisión de ILS en los distintos procedimientos judiciales, por mucho que estos estén cualificados y especializados en esta materia, no es en muchos casos suficiente para proporcionar a las personas sordas un acceso equitativo a los mismos. Es esencial que los diferentes profesionales del Derecho, ya sean jueces, abogados, fiscales, etc. tengan una formación mínima y suficiente sobre cómo trabajar con ILS y con personas sordas. Napier y Haug (2017) encuentran que solo el $24 \%$ de los encuestados afirma que en sus países europeos existe algún módulo específico en esta materia durante la formación universitaria de dichos profesionales, y obtienen ese mismo porcentaje cuando preguntan por la oferta de formación continua para estos sobre dicha temática mediante, por ejemplo, cursos o talleres. El reciente estudio de Pérez Senra (2019) nos permite conocer algunos aspectos de la realidad con la que se encuentran los ILS y los guías-intérpretes de personas sordociegas en salas de juzgados españolas debido a esta falta de formación por parte de los agentes implicados. Así, aunque no suele ser necesario justificar su presencia ante el tribunal como profesional de la interpretación, el $68 \%$ de las encuestadas afirma tener que dar explicaciones de la ubicación que deben asumir en la sala para poder desempeñar correctamente su función. Además, el 66,7\% "considera que los gestos propios de la comunicación en LS [lengua de señas] han sido valorados en alguna ocasión como indicadores de agresividad" (p. 81) por parte de las personas oyentes, quienes no comprenden que los componentes no manuales de las lenguas de señas son elementos esenciales en estas. Estas cuestiones, así como muchas otras, podrían fácilmente evitarse y los juicios serían más equitativos para las personas sordas si los profesionales del Derecho recibieran formación sobre el trabajo con estas, sus características lingüísticas y culturales y sobre cómo trabajar con ILS.

29. Ortega Herráez (2013) llevó a cabo un estudio sobre las expectativas en cuanto a calidad de la interpretación en ámbitos jurídicos que tienen las administraciones que subcontratan estos servicios. Para ello, realizó el análisis comparado de "un total de dieciséis pliegos técnicos que rigen este tipo de contratos en el ámbito de asilo, órganos jurisdiccionales y cuerpos policiales" (p. 15). Se centró en cuatro parámetros de calidad: la cualificación profesional de los intérpretes, su formación continua, la modalidad de interpretación empleada y la aplicación de la deontología profesional. Los resultados muestran que gran parte de los pliegos analizados recogían expresamente cuestiones relacionadas con dichos criterios de calidad. Sin embargo, el autor considera que esto no garantiza el efectivo cumplimiento de lo dispuesto en los mismos. Coincidimos con él en que es necesario - y urgente- que se establezcan "mecanismos de controt que permitan verficar el cumplimiento de lo que disponen" (ibid. p. 28) los pliesos de contratación a fin de garantizar en este ámbito servicios de calidad. 


\section{¿Cuántos intérpretes de lenguas de señas son necesarios en un proceso judicial y qué competencias deben poseer?}

Una diferencia importante que encontramos entre España y otros países, como Estados Unidos, Canadá u otros países europeos, es el número de ILS requeridos en un proceso judicial, lo que repercute en la calidad del propio servicio. Por regla general, en nuestro país solo se solicita y facilita un ILS, independientemente del número de personas sordas involucradas o de sus características lingüísticas y culturales, ya que se entiende que el ILS, como profesional formado que debe actuar de forma neutral e imparcial, puede realizar su labor sin dificultades en cualquier situación que se dé.

En Estados Unidos y Canadá se utiliza siempre un equipo compuesto por un mínimo de dos profesionales oyentes (RID, 2007; NCIEC³, 2009a; Russell, 2011; Roberson, Russell y Shaw, 2011). Por su parte, De Wit y Salami (2012) encuentran que, de los veintiocho países europeos analizados en su estudio, tan solo en Dinamarca, Estonia, Finlandia, Noruega, Suecia y Reino Unido es frecuente la participación de dos ILS en procedimientos judiciales. La complejidad de este tipo de discursos y las consecuencias de un posible error de interpretación para los usuarios y para la propia Administración de Justicia justifica el uso de equipos de interpretación de lengua de señas. Estos ILS se sustituyen entre sí normalmente cada treinta minutos, lo que evita la fatiga mental del profesional y se reduce así la cantidad de posibles errores. Además, permite que el ILS que está de apoyo realice una serie de actividades que mejoran la prestación del servicio y aseguran una interpretación adecuada y precisa, como por ejemplo la evaluación constante de su compañero en activo con el fin de detectar y evitar errores, asistirle con la toma de notas de datos concretos y estar atento a que las condiciones que se dan sean en todo momento las adecuadas para la interpretación (Roberson et al., 2011). Hale (2010) afirma que "trabajar en equipo con otro intérprete no solo mejoraría la salud y seguridad de ambos evitando una fatiga innecesaria, sino que también garantizaría la calidad del servicio" (p. 151), aunque aquí entra en juego el presupuesto destinado a labores de interpretación. En el documento que elaboró Russell (2011) — sobre la interpretación del discurso legal y cómo desarrollar el trabajo en este tipo de contexto- y en el del RID (2007) se enumeran una serie de situaciones en las que el número de miembros de equipos de interpretación puede aumentar, debido a la necesidad de que dos equipos de ILS independientes participen, e incluso a la posibilidad de que intervenga un ILS sordo. Los factores que determinan estas variaciones son la duración de los procesos, el número de personas sordas involucradas en el litigio, las habilidades requeridas por las características lingüísticas y culturales de los usuarios sordos, etc.

La participación de un ILS sordo como mediador entre el ILS oyente y el usuario sordo en entornos jurídicos es necesaria cuando este último presenta una baja competencia en la lengua de señas, ya sea por la edad (por ejemplo, en el caso de los niños y niñas) o forma en la que la adquirió, por la falta de acceso a una educación formal, o porque es una persona sorda extranjera competente en su lengua de señas, pero no en la del país receptor. Es frecuente además que estas personas desconozcan la figura del ILS y desconfíen de él, sintiéndose más cómodos comunicándose con otra persona sorda (De los Santos Rodríguez y Lara Burgos, 2004). Los ILS oyentes que trabajan en este tipo de contextos admiten que cuando trabajan con ILS

30. NCIEC son las siglas que corresponden al National Consortium of Interpreter Education Centers, fundado en 2006 por el Departamento de Educación de los Estados Unidos, que tiene como objetivo ampliar y mejorar la eficacia de los profesionales en interpretación mediante servicios de educación y desarrollo profesional y de los recursos que existen a nivel regional y nacional. Más información en http://www.interpretereducation.orq/, aunque la web está sin actualizar desde el 30 de septiembre de 2016. 
sordos mejora la precisión, sentido y eficacia de la interpretación jurídica y judicial (NCIEC, 2009a, p. 19). El NCIEC (2009b, pp. 9-10) señala que la intervención de un ILS sordo es efectiva cuando los usuarios sordos presentan algunas de las siguientes características:

- Competencia lingüística limitada en la lengua de señas.

- Una socialización limitada en la comunidad sorda.

- Nivel educativo insuficiente.

- Dificultades cognitivas.

- Retraso en el lenguaje.

- Problemas orgánicos que causan otras deficiencias.

- Enfermedad mental.

- Problemas causados por el abuso de drogas.

- Otros problemas físicos.

El RID (2007) afirma que "(l)ong years of experience have demonstrated that native deaf users of ASL [American Sign Language] are more effective at communicating with this segment of the population that the general practitioner interpreter who can hear" (p. 2).

Otro grupo poblacional que se beneficia del ILS sordo en juzgados o contextos legales es el de menores sordos. Diversos factores, tales como la edad, el tipo y nivel educativo alcanzado, el tiempo y calidad de exposición a la lengua de señas, la experiencia previa de comunicarse a través de un ILS, su estado emocional, etc., pueden hacer que el menor no presente las capacidades lingüísticas y cognitivas suficientes que le permitan participar con plenitud en una comunicación mediada por un ILS oyente en el ámbito jurídico (NCIEC, 2009a, p. 21). El NCIEC (2009b) va más allá y plantea un modelo alternativo al tradicional de interpretación para cualquier persona sorda en ámbito jurídico: un equipo de interpretación sordo-oyente ${ }^{3}$. Utiliza numerosos procedimientos judiciales como ejemplos para justificar la necesidad de contar con ILS sordos en juzgados, además de su hipótesis de que ese modelo:

to protect the rights of deaf litigants is more inclusive and efficient than the typical spoken language interpreter model. Deaf interpreters, assisted by court interpreters who can hear, are the reasonable accommodation that ensures that most deaf litigants, not just those with specific language challenges, are afforded full and equal access to justice. (NCIEC, 2009b, p. 7).

Es más, plantea que en muchos casos el intérprete principal en el juicio debe ser el ILS sordo, mientras que el ILS oyente actúa solo como su intérprete o personal adjunto (cfr. 2009b, p. 38). Pero para que este tipo de adaptación sea realmente efectiva, el ILS sordo debe ser una persona con una formación y habilidades concretas. No cabe la sugerencia que hacen De los Santos Rodríguez y Lara Burgos (2004) de "valerse de la colaboración de una persona sorda como 'intérprete'" (p. 163), sino que se debería seguir el modelo utilizado en Estados Unidos en el que el ILS sordo es un profesional que también ha tenido que pasar por un proceso de formación y certificación de competencias ${ }^{32}$.

31. EL NCIEC (2011) publicó un material audiovisual en el que se explica cómo se trabaja en equipo con un ILS sordo en el ámbito jurídico. Se trata de un juicio simulado en el que un equipo de intérpretes sordo-oyente asisten a una persona sorda que actúa como testigo. En el siguiente enlace se puede visualizar el juicio completo: https://www.youtube.com/watch?v=699ionfzzVMyindex=4ylist=PLeltAHhzEgYnj4DuZ-ffmm1MQ4gD-biSs

32. El RID certifica desde 1998 como ILS a aquellas personas sordas o hipoacúsicas que, tras pasar por una formación de 40 horas y una serie de exámenes teórico-prácticos, demuestran poseer una serie de conocimientos sobre interpretación, sordera, cultura y comunidad sorda, así como habilidades lingüísticas en ASL y en otros recursos comunicativos. Información disponible en: http://www.rid.org/rid-certification-overview/cdi-certification/ 
En Estados Unidos también encontramos que, además del habitual equipo de dos ILS costeado por la Administración y funcionarios del juzgado (RID, 2007; Napier et al., 2010), hay juicios en los que puede participar hasta un tercer ILS, como miembro del equipo defensor de una persona sorda. A dicho ILS lo contrata de forma privada esta parte y asiste al juicio para facilitar la comunicación entre el usuario sordo y su abogado durante la celebración de este. Además, controla que la interpretación que está haciendo el equipo de ILS es adecuada, lo que "enables counsel to interpose objections to the interpretation immediately to preserve the right to appeal based on a faulty interpretation" (RID, 2007, p. 3).

Por lo tanto, como hemos visto -y a diferencia de lo que ocurre en nuestro país, en el que predomina el paradigma tradicional de un solo ILS oyente como ajuste razonable para el acceso a la información y a la comunicación en este ámbito-, no existe un número determinado de ILS para un proceso judicial, sino que cada caso es particular y desde la Administración de Justicia se debe valorar con anterioridad cuántos son los profesionales requeridos, así como sus competencias y habilidades, teniendo en cuenta las necesidades y características de las personas sordas, para que estas puedan participar de forma equitativa y plena en el mismo.

\section{Conclusiones}

Aunque mediante fórmulas de externalización de los servicios por parte de la Administración pública, el número de servicios de interpretación de lengua de señas en España para los servicios públicos ha aumentado en estos años, tanto de forma presencia como a distancia. Esto refleja el grado de accesibilidad y participación en la sociedad que la comunidad sorda ha ganado en las últimas décadas. Además, la presencial de estos profesionales en los contextos jurídicos es amplia y abarca diferentes escenarios. Esta asistencia lingüística está garantizada por nuestro ordenamiento jurídico, que desarrolla los derechos constitucionales que amparan a cualquier ciudadano español. La publicación de la Ley 27/2007 supuso un antes y un después para la comunidad sorda en cuanto al disfrute de sus derechos y uso de la lengua de señas en los diferentes contextos sociales mediante ILS. Pero es la promulgación de la Ley Orgánica 5/2015, con la trasposición a nuestro ordenamiento de las directivas europeas en materia de traducción e interpretación, la que asegura la protección jurídica de los derechos de este colectivo para disfrutar de procedimientos judiciales equitativos y una tutela judicial efectiva.

No obstante, tras la revisión de la Ley Orgánica 5/2015 para la interpretación de lengua de señas, y gracias al análisis que hemos hecho valorando cómo es la dotación de estos servicios en otros países europeos y del mundo en los que esta profesión tiene mayor desarrollo y experiencia, consideramos que es esencial que se tengan en cuenta y se modifiquen, en la medida de lo posible, algunas cuestiones vinculadas con la provisión de estos servicios por parte de la Administración de Justicia española, en pro de ofrecer a los usuarios sordos una mayor calidad en los mismos. En primer lugar, es esencial incluir a los traductores e intérpretes de lenguas de señas españolas en el Registro Oficial de Traductores e Intérpretes Judiciales, una vez este se ponga en funcionamiento, y que estas lenguas viso-gestuales también sean objeto de valoración en los exámenes conducentes al título de Intérprete y Traductor Jurado. Solo mediante la evaluación de competencias específicas y la existencia de sistemas de acreditación periódica de los ILS que desempeñan su función en estos contextos se puede asegurar una labor que ofrezca garantías suficientes de exactitud y, por tanto, evitar situaciones en las que se solicite la designación de otro ILS, con lo que esto supone para el normal desarrollo de los procedimientos judiciales 
y para la propia profesión de ILS. Intrínsecamente vinculada a esta cuestión está la fórmula de contratación de estos servicios y la contraprestación económica que reciben los ILS, teniendo en cuenta la trascendencia que tiene su labor en la vida de las personas involucradas. Abogamos porque tanto el Ministerio de Justicia como el Ministerio del Interior eviten la subcontración de estos servicios a empresas privadas, pues supone un mayor coste para las arcas públicas y una disminución de la calidad del servicio, y apuesten por la convocatoria de oposiciones a funcionarios como, por ejemplo, hace el MAEC para el Cuerpo de Traductores e Intérpretes del Estado.

Otro aspecto que se ha vislumbrado en este trabajo es la importancia que tiene la especialización de los ILS en materia jurídica y la formación de estudiantes y profesionales del Derecho en cuestiones básicas sobre cómo trabajar con estos y con personas sordas. Tanto en la enseñanza universitaria de futuros ILS como en la oferta de formación continua a los profesionales ya titulados se deben incluir contenidos teóricos y prácticos que favorezcan su desempeño en contextos jurídicos, haciendo especial hincapié en el análisis de estas situaciones comunicativas, de los roles que asumen los participantes y de la diferencia de poder relativo que existe entre ellos, del lenguaje jurídico, de los tipos de textos y su equivalencia en las lenguas de señas, de las técnicas y estrategias de interpretación más adecuadas en cada caso, etc. Por último, para poder garantizar una prestación de servicios de interpretación con precisión y rigor, adaptada a las necesidades comunicativas, lingüísticas y cognitivas de cada una de las personas que componen la comunidad sorda, es primordial que, en los distintos procedimientos judiciales, empiecen a ser frecuentes los equipos compuestos, como mínimo, por dos ILS, y que incluso uno de ellos sea un profesional sordo adecuadamente formado.

\section{Bibliografía}

Acosta Rodríguez, V. (2006). Los problemas de las personas sordas para su integración en el mundo laboral. Análisis de la realidad y propuestas de acción. Innovación Educativa, (16), 257-273. Recuperado de http://minerva.usc.es/bitstream/10347/4397/1/pg 257-274 inneduc16.pdf

Báez Montero, I. C. y Fernández Soneira, A. M. (2015). Historia de la formación de los intérpretes de LSE en España. En I. C. Báez Montero, I. C. y H. Otero Doval (Eds.), Buscando respuestas en lengua de signos. Experiencias docentes con LSE como base de enseñanza (pp.125-131). Lugo, España: Axac.

Bao Fente, M. y González-Montesino, R. H. (2013). Aproximación a los parámetros de calidad en la interpretación de la lengua de signos española. En R. Barranco-Droege, E. M. Pradas Macías y 0. García Becerra (Eds.), Quality in interpreting: widening the scope Vol. 2. (pp. 293-314). Granada, España: Comares.

Belda Pérez-Pedrero, E. (2016). Las lenguas de signos españolas. Su necesaria presencia en el debate social y doctrinal sobre la reforma de la Constitución española. Revista de Derecho Político, (96). 87-120. doi: https://doi.org/10.5944/ rdp.96.2016.17055

Brunson, J. L. (2008). Your Case Will Now Be Heard: Sign Language Interpreters as Problematic Accommodations in Legal Interactions. Journal of Deaf Studies and Deaf Education, 13(1), 77-91. doi: http://dx.doi.org/10.1093/deafed/enm032

Confederación Estatal de Personas Sordas. (2003). Libro blanco de la lengua de signos en el sistema educativo. Madrid, España: Confederación Estatal de Personas Sordas.

Confederación Estatal de Personas Sordas. (2012). Menos servicios de intérpretes para las personas sordas. Faro del silencio, (240). 26-32. Recuperado de http:// www.cnse.es/uploaded/publicaciones/240.pdf 
Confederación Estatal de Personas Sordas. (s.f.): Memoria 2017. Madrid: CNSE. Recuperado de http://www.cnse.es/uploaded/publicaciones/21032019081338 3790. $\underline{\mathrm{pdf}}$

Confederación Estatal de Personas Sordas. (s.f.): Memoria 2018. Madrid: CNSE. Recuperado de http://www.cnse.es/uploaded/publicaciones/140220201345169325. pdf

De los Santos Rodríguez, E. y Lara Burgos, M. P. (2004). Técnicas de interpretación de lengua de signos. (2 ${ }^{\mathrm{a}}$ ed.). Madrid, España: Fundación CNSE.

De Wit, M. y Salami, M. (2012). Sign language interpreting in legal settings: new scenarios within the European legal framework and the efsli experience. En W. Baur, B. Eichner, S. Kalina, y F. Mayer (Eds.), Übersetzen in die Zukunft (pp. 490-496). Berlín, Alemania: BDÜ Fachverlag.

Gascón Nasarre, F. A. (2011, diciembre). Una breve radiografía de la interpretación judicial en España. La Linterna del Traductor, (6). 31-40. Recuperado de http://www. lalinternadeltraductor.org/pdf/lalinterna n6.pdf

Hale, S. (2010): La interpretación comunitaria: la interpretación en los sectores jurídico, sanitario y social. (C. Valero Garcés y R. Cobas, trad.). Granada, España: Comares.

Ibáñez Castaño, A. (2019). Materia legislativa relativa al derecho a la interpretación y a la traducción en los procesos penales para las personas sordas. Revista de la Facultad de Derecho de México, 274(2), 727-752. doi: http://dx.doi.org/10.22201/ fder.24488933e.2019.274-2.70040

Napier, J. (2004). Sign language interpreter training, testing and accreditation: An international comparison. American Annals of the Deaf, 149(4), 350-360. doi: http:// dx.doi.org/10.1353/aad.2005.0007

Napier, J. (2013). Legal Interpreting, Deaf people and jury service...a happy union? NEWSLI: Magazine of the Association of Sign Language Interpreters of England, Wales and Northern Ireland, 6-12. Recuperado de https://www.academia.edu/7069181/ Legal interpreting deaf peple and jury service

Napier, J. y Haug, T. (2017). Justisigns: A European overview of sign language interpreting provision in legal settings. Law, Social Justice and Global Development 2016(2).

Napier, J., McKee, R. L. y Goswell, D. (2010). Sign language interpreting: Theory and practice in Australia and New Zealand. Annandale, N.S.W: Federation Press.

Napier, J., Spencer, D. y Sabolcec, J. (2007). Deaf jurors' access to court proceedings via sign language interpreting: an investigation. Research Report 14. Sydney, N.S.W: New South Wales Law Reform Commission. Recuperado de https://www.lawreform. justice.nsw.gov.au/Documents/Publications/Other-Publications/Research-Reports/RR14.pdf

National Consortium of Interpreter Education Centers (2009a). Best Practices American Sign Language and English Interpretation Within Legal Settings. [s.l.]: NCIEC - Legal Interpreting Workgroup. Recuperado de http://www.interpretereducation.org/ wp-content/uploads/2011/06/LegalBestPractices NCIEC2009.pdf

National Consortium of Interpreter Education Centers (2009b). Deaf Interpreters in Court: An accommodation that is more than reasonable. [s.l.]: NCIEC - Legal Interpreting Workgroup. Recuperado de http://www.interpretereducation.org/wp-content/ uploads/2011/06/Deaf-Interpreter-in-Court NCIEC2009.pdf

Ortega Herráez, J. M. (2011). Cómo acreditar intérpretes a través de la traducción: análisis crítico de la acreditación profesional de intérpretes jurídicos en España. TRANS. Revista de traductología, (15), 131-153. Recuperado de http://www.trans. uma.es/pdf/Trans 15/131-153.pdf 
Ortega Herráez, J. M. (2013). La intérprete no sólo tradujo lo que le vino en gana, sino que respondió ella a las preguntas que los abogados le realizaban al testigo: requisitos de calidad en la subcontratación de servicios de interpretación judicial y policial en España. Sendebar, (24), 9-42.

Pérez Senra, B. (2019). La interpretación judicial en lengua de signos: una cuestión de rol. La percepción de las intérpretes de lengua de signos de la Comunidad Valenciana de su rol ante el tribunal. Revista de Llengua i Dret, Journal of Language and Law, (71), 73-87. doi: http://dx.doi.org/10.2436/rld.i71.2019.3300

Pöchhacker, F. (2004). Introducing interpreting studies. London, Great Britain: Routledge. Red2Red Consultores (2009). Discapacidad, estudios superiores y mercado laboral. Barreras de acceso y repercusión en la inserción laboral. Madrid, España: Fundación ONCE

Registry of Interpreters for the Deaf [RID]. (2007). Interpreting in Legal Settings. Recuperado de http://www.rid.org/about-interpreting/standard-practice-papers/

Roberson, L., Russell, D. y Shaw, R. (2011). American Sign Language/English Interpreting in Legal Settings: Current Practices in North America. Journal of Interpretation, 21(1), 64-79. Recuperado de http://digitalcommons.unf.edu/cgi/viewcontent. cgi?article $=1000 \&$ context $=$ joi

Russell, D. (2011). Interpreting legal discourse \& working in legal settings: an AVLIC position paper. [s.l.]: Association of Visual Language Interpreters of Canada. Recuperado de http://www.avlic.ca/sites/default/files/docs/AVLIC-Interpreting Legal Discourse $\% 26$ Working in Legal Settings.pdf

Sarlo, 0. (2006). El marco teórico en la investigación dogmática. En C. Courtis. (Ed.) Observar la ley: ensayos sobre metodología de la investigación jurídica (pp. 175-208). Colección Estructuras y Procesos, Serie Derecho. Madrid, España: Trotta.

Storch de Gracia y Asensio, J. G. (1998). El nombre de nuestra lengua. Reflexiones acerca de la polémica creada sobre la denominación de "lengua gestual", "lengua de señas" o "lengua de signos". Comunicación presentada al I Congreso Ibero-Americano de Educación Bilingüe para Sordos. Lisboa, 6-10 de julio de 1998. Recuperado de https://webs.ucm.es/info/civil/bardecom/docs/signa.pdf

Storch de Gracia y Asensio, J. G. (2008). Las lenguas de señas ante el Derecho civil. En Revista General de Legislación y Jurisprudencia, (2), 259-302.

Tantaleán Odar, R. M. (2016). Tipología de las investigaciones jurídicas. Derecho y Cambio Social, 13(46), 1-37. 